\title{
Extended Research on Grey Target Decisions for a Variable Target Centre Based on Decision Maker Preferences
}

\author{
Hongna Song, Xinxian Zhai, and Jinshan Ma \\ School of Energy Science and Engineering, Henan Polytechnic University, Jiaozuo, Henan 454000, China \\ Correspondence should be addressed to Jinshan Ma; majins5@163.com
}

Received 5 July 2019; Revised 18 September 2019; Accepted 17 October 2019; Published 14 November 2019

Academic Editor: Emilio Jiménez Macías

Copyright (C) 2019 Hongna Song et al. This is an open access article distributed under the Creative Commons Attribution License, which permits unrestricted use, distribution, and reproduction in any medium, provided the original work is properly cited.

\begin{abstract}
The impact of target centre indices determined by the desires and selection preferences of decision makers (DMs) on target centre distances (TCDs) has been discussed extensively. One way of facilitating this is by using a multiple-attribute grey target decision method (GTDM), where normalisation plays an important role. So far, however, TCDs have only undergone linear normalisation. Therefore, we investigated the available normalisation methods with regard to the impact of a variable target centre on TCDs and objective weights. Our work shows that existing normalisation methods can be divided into two kinds: those that affect the index TCDs and determine the objective weights and those that do not, with target centre indices instead determined by the preferences of DMs. Finally, numerical examples are provided and applications are discussed.
\end{abstract}

\section{Introduction}

The grey target decision method (GTDM) established by Deng [1] is widely used, owing to its simplicity and because decisions can be made exclusively based on the alternatives themselves. The method ranks alternatives by the target centre distance (TCD) - the distance from an alternative to its target centre-where the smaller the value, the better the choice. TCDs are determined by comparing all the alternatives under all attributes to seek the best values. Many researchers have advanced the method in various respects. Dang studied how weights are determined [2-4]. Zhu and Hipel extended the GTDM with attribute values handled from only real numbers to other data types such as fuzzy numbers and linguistic numbers [4-10]. Ma proposed a mixed-attribute GTDM with attribute weights containing uncertain numbers [11]. Song et al. proposed a way of contributing to group decisions with the GTDM [12, 13]. Furthermore, other theories and methods have adapted the GTDM [14-18]. Ma and Ji proposed a generalised grey target decision method (GGTDM) [8, 19], and a mixed-attribute GGTDM was developed based on the Kullback-Leibler distance and the Gini-Simpson index [20-22]. Ma studied how index TCDs are affected by a variable target centre determined by the desires or selection preferences of a decision maker (DM) with multiple-attribute decisionmaking (MADM) [19]. Subsequently, Ma and Sun [23] extended the research in [19] by introducing a negative target centre.

The proof in [19] shows that the positive target centre determined by DM's different preferences affects the index TCDs. The target centre index decided by DM's desires can narrow the difference between the index TCDs, while the target centre determined by DM's selection preferences can enlarge the difference between the index TCDs. Nevertheless, some index TCDs are regarded as equally excellent-and, as such, are no less than their target centre index. The target centre decided by the DM's selection preferences has the function of "rewarding good and punishing bad." However, [23] advances [19] by expanding the positive target centre to the negative target centre with regard to the DM's desires and selection preferences. Furthermore, the research concludes that a positive or negative target centre decided by a DM's desires can narrow the difference between the index TCDs-referred to as clustering the index TCDs for all alternatives. The function of "rewarding good and punishing bad" means that some index TCDs are no different when they are 
superior to the positive target centre value, and some index TCDs have a larger gap when they are inferior to the positive target centre value. The impact of target centre indices determined by a DM's preferences on index TCDs can be ascribed to the normalisation process or a dimensionless process. Indeed, normalisation plays a pivotal role in MADM. Research on this issue focuses on different aspects. $\mathrm{Li}$ et al. studied the principles of choosing dimensionless methods in terms of their variability, diversity, and stability [24]. Zhan et al. analysed the character of linear dimensionless methods and proposed that various dimensionless methods can be used in MADM at the same time [25]. Liu researched a nonlinear normalisation method for special purposes [26]. Jahan and Edwards reviewed the state-of-the-art normalisation techniques in the materials-selection process for engineering design [27]. Due to the importance of the Technique for Order Preference by Similarity to Ideal Solution (TOPSIS) method, the impact of normalisation methods on this method has been studied by some researchers $[28,29]$.

However, the variable target centre decided by a DM's preferences proposed by $[8,19]$ only considers a linear method of normalising the TCDs. Normalisation affects TCDs in different ways by changing the target centre. Therefore, the aim of our study is to determine whether other normalisation methods can also reach the same conclusions as those in $[19,23]$. Based on the data in [19], we considered several normalisation methods, including the min-max normalisation method, the vector normalisation method, and the linear proportional normalisation method. Furthermore, we studied the effect of different normalisation methods on objective weights.

The contributions of this research are as follows. We uncover which commonly used normalisation methods for target centre indices determined by DM preferences affect the index TCDs and objective weights, and which ones do not. Furthermore, we explain the reasons why a given normalisation method may or not affect index TCDs and objective weights.

Our research focuses on the GTDM. However, we expect that our findings will also be helpful to other MADA methods, such as TOPSIS, Grey Relational Analysis (GRA), and Extension Theory [30-32]. Indeed, the various data handled by most MADA methods must be normalised for a proper comparison. In general, our research shows that the choice of the normalisation method can impact the results of decision-making, which is an interesting issue.

\section{Preliminaries}

2.1. Normalisation Methods. It is important to normalise different indices for comparisons in MADA [33]. Some typical normalisation methods are given as follows [34].

2.1.1. Min-Max Normalisation Method. The min-max normalisation method consists of equations (1)-(3):

$$
\begin{aligned}
y_{i j} & =\frac{x_{i j}-m_{j}}{M_{j}-m_{j}}, \quad i=1,2, \ldots, n ; j=, 2, \ldots, m, \\
y_{i j} & =\frac{M_{j}-x_{i j}}{M_{j}-m_{j}}, \quad i=1,2, \ldots, n ; j=, 2, \ldots, m, \\
M_{j} & =\max _{i}\left\{x_{i j}\right\}, \\
m_{j} & =\min _{i}\left\{x_{i j}\right\},
\end{aligned}
$$

where equations (1) and (2) are for a benefit- and a cost-type attribute, respectively; $x_{i j}$ is a non-normalised index value; $y_{i j}$ is the normalised index value; and $M_{j}$ and $m_{j}$ are the maximum value and minimum value under attribute $A_{j}$, respectively.

2.1.2. Vector Normalisation Method. Equation (4) shows the vector normalisation method:

$$
y_{i j}=\frac{x_{i j}}{\sqrt{\sum_{i=1}^{n} x_{i j}^{2}}}, \quad i=1,2, \ldots, n ; j=1,2, \ldots, m,
$$

where $x_{i j}$ is a non-normalised index value and $y_{i j}$ is the normalised index value.

2.1.3. Linear Proportional Normalisation Method. The following equation is referred to as the linear proportional (LP) normalisation method:

$$
y_{i j}=\frac{x_{i j}}{M_{j}}, \quad i=1,2, \ldots, n ; j=1,2, \ldots, m,
$$

where $M_{j}$ is a special point that may be set as the maximum value, minimum value, or mean value under attribute $A_{j}$. In this research, $M_{j}$ is given by equation (3).

2.1.4. Linear Normalisation Method. Equation (6) shows the linear normalisation method:

$$
y_{i j}=\frac{x_{i j}}{\sum_{i=1}^{n} x_{i j}}, \quad i=1,2, \ldots, n ; j=1,2, \ldots, m,
$$

where $\quad x_{i j} \geq 0, \quad \sum_{i=1}^{n} x_{i j}>0(i=1,2, \ldots, n ; j=1,2, \ldots, m)$ and $y_{i j}$ is the normalised index value.

2.2. Method of Determining Objective Weights. Subjective weights and objective weights are the two kinds of basic weights determined by different mechanisms. Many researchers have studied how weights are determined. Subjective weights depend on DM preferences, whereas objective ones originate from the alternatives themselves through different mechanisms. In this study, we exclusively consider objective weights, in order to determine whether different normalisation methods affect them.

The weights of all attributes can be determined objectively by the deviation of the normalised TCDs. The objective weight model is as follows [8]. 
For any two alternatives $C_{s}$ and $C_{t}$, under attribute $A_{j}$, the deviation of index values $z_{s t j}=\left|z_{s j}-z_{t j}\right|$. Thus, all the deviations of all alternatives under attribute $A_{j}$ are derived as follows:

$$
z_{j}=\sum_{s=1}^{n-1} \sum_{t=s+1}^{n} z_{s t j}=\sum_{s=1}^{n-1} \sum_{t=s+1}^{n}\left|z_{s j}-z_{t j}\right|
$$

The attribute weights can be obtained by maximizing all the deviations, such that the following model can be constructed:

$$
\max f=\sum_{j=1}^{m} z_{j} \omega_{j}^{1 / 2} \sum_{j=1}^{m} \omega_{j}=1, \omega_{j} \geq 0, \quad j=1, \ldots, m .
$$

To solve the nonlinear program (8) via the Lagrange function, the weights are obtained as follows:

$$
\omega_{j}=\frac{z_{j}^{2}}{\sum_{j=1}^{m} z_{j}^{2}}, \quad j=1,2, \ldots, m,
$$

where $\omega_{j}$ is the weight of attribute $A_{j}$.

\subsection{Generalised Grey Target Decision Method}

2.3.1. Basic Concepts. Definition 1. Suppose an MADA problem and let $S=\left(S_{1}, S_{2}, \ldots, S_{n}\right)$ and $A=\left(A_{1}\right.$, $\left.A_{2}, \ldots, A_{m}\right)$ be the alternative set and attribute set, respectively. The measure of $S_{i}$ on $A_{j}$ is $S_{i j}(i=1,2, \ldots, n, j=$ $1,2, \ldots, m)$ with the benefit-type attribute set and cost-type attribute set denoted by $J^{+}$and $J^{-}$, respectively.

Definition 2. The GGTDM is based on the principle of the GTDM but differs from it in two ways: there is no need to normalise the measure $S_{i j}(i=1,2, \ldots, n, j=1,2, \ldots, m)$ in advance; and the target centre distance is calculated differently.

Definition 3. The target centre determined by the measure $S_{i j}(i=1,2, \ldots, n, j=1,2, \ldots, m)$ is $C^{a}=\left(C_{1}^{a}, C_{2}^{a}, \ldots, C_{m}^{a}\right)$, where $C_{j}^{a}(j=1,2, \ldots, m)$ satisfies

$$
C_{j}^{a}=\left\{\begin{array}{l}
\max \left\{S_{i j}\right\}, S_{i j} \in J^{+}, \\
\min \left\{S_{i j}\right\}, S_{i j} \in J^{-},
\end{array} \quad i=1,2, \ldots, n, j=1,2, \ldots, m .\right.
$$

Definition 4. The desirable preference value $C_{k}^{d e}$ $(k \in\{1,2, \ldots, m\})$ is such that the DM hopes the value is superior to the best value of all alternatives under some attribute that satisfies

$$
\begin{aligned}
C_{k}^{d e} & \geq \max \left\{S_{i j}\right\}, S_{i j} \in J^{+}, \quad i=1,2, \ldots, n, j=1,2, \ldots, m \\
\text { or } C_{k}^{d e} & \leq \min \left\{S_{i j}\right\}, S_{i j} \in J^{-}, \quad i=1,2, \ldots, n, j=1,2, \ldots, m .
\end{aligned}
$$

Definition 5. The selection preference value $C_{k}^{d s}(k \in$ $\{1,2, \ldots, m\})$ is such that the DM thinks the value is apt if it reaches a certain value under some attribute that satisfies

$$
\min \left\{S_{i j}\right\}<C_{k}^{d s}<\max \left\{S_{i j}\right\}, \quad i=1,2, \ldots, n, j=1,2, \ldots, m .
$$

Definition 6. Let $C^{a}=\left(C_{1}^{a}, C_{2}^{a}, \ldots, C_{m}^{a}\right)$ be the target centre vector determined by $S_{i j},(i=1,2, \ldots, n, j=1,2, \ldots, m)$, and let the DM preference value be $C_{k}^{d}(k \in\{1,2, \ldots, m\})$ under attribute $A_{k}(k \in\{1,2, \ldots, m\})$. Then, the final target centre vector combined with the DM preference values is $C^{0}=\left(C_{1}^{0}, C_{2}^{0}, \ldots, C_{m}^{0}\right)$, where $C_{j}^{0}(k=1,2, \ldots, m)$ satisfies

$$
C_{j}^{0}= \begin{cases}C_{j}^{a}, & j=1,2, \ldots, m, j \neq k \\ C_{k}^{d}, & k \in\{1,2, \ldots, m\}, j=k\end{cases}
$$

2.3.2. Calculating the Target Centre Distance [19]. Suppose that $C^{0}=\left(C_{1}^{0}, C_{2}^{0}, \ldots, C_{3}^{0}\right)$ is the target centre vector partially determined by DM preferences from the measure $S_{i j}(i=1,2, \ldots, n, j=1,2, \ldots, m)$. The TCDs are then obtained by the following steps.

(1) The new measure $I_{i j}$ derives from comparing the measure $S_{i j}(i=1,2, \ldots, n, j=1,2, \ldots, m)$ with $C_{j}^{0}(j=1,2, \ldots, m) \quad$ under attribute $A_{j}(j=$ $1,2, \ldots, m)$ using (14).

$I_{i j}=\left\{\begin{array}{l}S_{i j}, \text { if }\left(S_{i j}<C_{j}^{0} \text { and } S_{i j} \in J^{+}\right) \text {or }\left(S_{i j}>C_{j}^{0} \text { and } S_{i j} \in J^{-}\right), \\ C_{j}^{0}, \text { if }\left(S_{i j} \geq C_{j}^{0} \text { and } S_{i j} \in J^{+}\right) \text {or }\left(S_{i j} \leq C_{j}^{0} \text { and } S_{i j} \in J^{-}\right) .\end{array}\right.$

(2) The index TCD of $I_{i j}(i=1,2, \ldots, n, j=1,2, \ldots, m)$ to $C_{j}^{0}(j=1,2, \ldots, m)$ is calculated using $(15)$ :

$r_{i j}=\left|C_{j}^{0}-I_{i j}\right|, \quad i=1,2, \ldots, n, j=1,2, \ldots, m$.

(3) The original index TCD $r_{i j}$ is normalised for comparison under attribute $A_{j}$. The linear method is used to normalise the index TCD using (16):

$z_{i j}=\frac{r_{i j}}{\sum_{i=1}^{n} r_{i j}}, \quad i=1, \ldots, n ; j=1, \ldots, m$,

where $z_{i j}$ is the normalised TCD.

(4) Given the weight $\omega_{j}$ under attribute $A_{j}(j=1$, $2, \ldots, m)$, the integrated TCD is obtained using (17):

$$
w_{i}=\sum_{j=1}^{m} \omega_{j} z_{i j}, \quad i=1, \ldots, n .
$$

2.3.3. Decision-Making Process. The algorithm for the proposed method is shown in Figure 1 and given as follows. It should be noted that the symbols that appear in the following steps are discussed in the definitions above. 


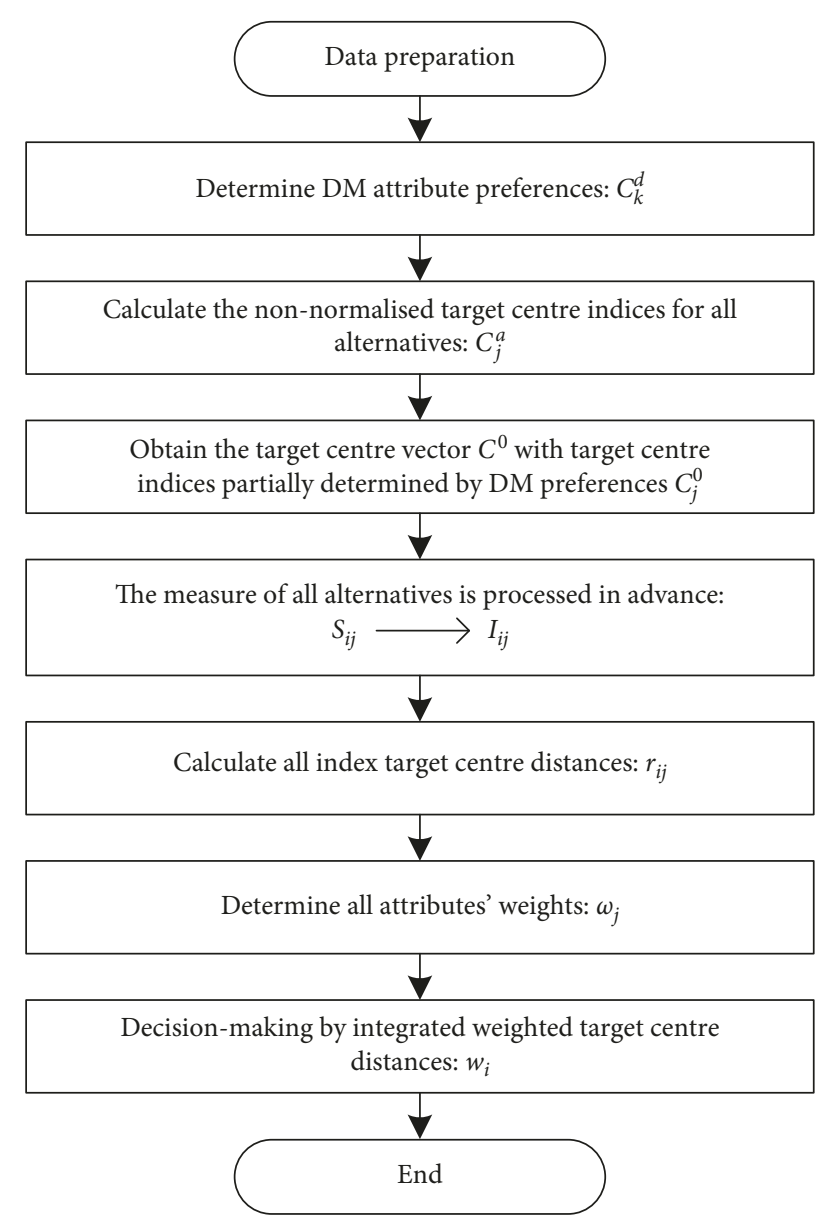

FIGURE 1: Flowchart of the proposed method.

(1) Determine the DM attribute preferences $C_{k}^{d}(k \in\{1,2, \ldots, m\})$.

(2) Calculate the non-normalised target centre indices $C_{j}^{a}(j=1,2, \ldots, m)$ for all alternatives.

(3) Obtain the target centre vector $C^{0}=\left(C_{1}^{0}, C_{2}^{0}, \ldots, C_{3}^{0}\right)$ with target centre indices partially determined by DM preferences $C_{j}^{0}(k=1,2, \ldots, m)$.

(4) The measure of all alternatives $S_{i j}(i=1,2, \ldots, n, j=$ $1,2, \ldots, m)$ is transformed into $I_{i j}(i=1,2, \ldots, n$, $j=1,2, \ldots, m)$ in advance.

(5) Calculate all index TCDs of $I_{i j}(i=1,2, \ldots, n, j=$ $1,2, \ldots, m)$ to $C_{j}^{0}(j=1,2, \ldots, m)$, denoted by $r_{i j}$.

(6) Determine all the objective weights of the attributes, $\omega_{j}(j=1,2, \ldots, m)$.

(7) Calculate the integrated,weighted TCDs $w_{i}(i=$ $1,2, \ldots, n)$ and make decisions according to the integrated TCD value of each alternative with which the smaller value being the better.

\section{Impact of Different Normalisation Methods on TCDs and Objective Weights}

3.1. Basic Variables and Parameters. The impact of the target centre determined different DM preferences on index TCDs is analysed as follows. Let $S_{i j}(i=1,2, \ldots, n, j=1,2, \ldots, m)$ be the measure of $S_{i}$ under attribute $A_{j}$. Let $d_{i_{0}}$ and $d_{i_{0}+1}$ be the distance from indices $S_{i_{0} j}$ and $S_{\left(i_{0}+1\right) j}$ to the target centre $C_{j}^{a}$, respectively, with $d_{i_{0}}<d_{i_{0}+1}$. Still, $D_{i_{0}}$ and $D_{i_{0}+1}$ denote the distance from $S_{i_{0} j}$ and $S_{\left(i_{0}+1\right) j}$ to the target centre $C_{j}^{d}$, respectively, determined by DM preferences under attribute $A_{j}$. And $r_{\mathrm{da}}$ is the distance from $C_{j}^{d}$ to $C_{j}^{a}$.

3.2. Effect of Different Normalisation Methods on Target Centre Distance. The impact of the linear normalisation method on index TCDs was studied in [19]. Here, we adopt some typical methods with the same parameters: the min-max normalisation method, the vector normalisation method, and the linear proportional normalisation method.

3.2.1. Min-Max Normalisation Method. The normalised difference of two TCDs with the min-max normalisation method regarding a different target centre is obtained using (18) according to the above situation:

$$
\Delta Z_{a}=\frac{d_{i_{0}+1}-m_{d}}{M_{d}-m_{d}}-\frac{d_{i_{0}}-m_{d}}{M_{d}-m_{d}}=\frac{d_{i_{0}+1}-d_{i_{0}}}{M_{d}-m_{d}},
$$

where $d_{i}$ is the distance from $S_{i j}$ to $C_{j}^{a}$ under attribute $A_{j}$, calculated by $d_{i}=\left|S_{i j}-C_{j}^{a}\right|$.

$$
\Delta Z_{d}=\frac{D_{i_{0}+1}-m_{D}}{M_{D}-m_{D}}-\frac{D_{i_{0}}-m_{D}}{M_{D}-m_{D}}=\frac{D_{i_{0}+1}-D_{i_{0}}}{M_{D}-m_{D}},
$$

where $D_{i}$ is the distance from $S_{i j}$ to $C_{j}^{d}$ under attribute $A_{j}$, calculated by $D_{i}=\left|S_{i j}-C_{j}^{d}\right|$.

The target centre index decided by the alternative has the relationship with the target centre determined by DM preferences as follows:

$$
r_{d a}=C_{j}^{d}-C_{j}^{a}
$$

Equation (19) can be converted into (21) according to (20):

$$
\Delta Z_{d}=\frac{\left(d_{i_{0}+1}+r_{d a}\right)-\left(d_{i_{0}}+r_{d a}\right)}{M_{D}-m_{D}}=\frac{d_{i_{0}+1}-d_{i_{0}}}{M_{D}-m_{D}} .
$$

From the above equations, we continue our analysis as follows:

(1) If $r_{d a}>0$, the target centre is decided by DM desires. In this case, the equality $M_{D}-m_{D}=M_{d}-m_{d}$ can be obtained by $M_{d}=\max _{i}\left\{d_{i}\right\}, m_{d}=\min _{i}\left\{d_{i}\right\}, M_{D}=$ $\max _{i}\left\{d_{i}+r_{d a}\right\}$, and $m_{D}=\min _{i}\left\{d_{i}+r_{d a}\right\}$.

Then, the equality $\Delta Z_{a}=\Delta Z_{d}$ holds when comparing equations (18) and (21). Thus, the normalised difference of the two TCDs remains unchanged. Consequently, there is no impact on index TCDs when the target centre is determined by DM desires.

(2) If $r_{d a}<0$, the target centre is decided by DM selection preferences, and the above equality does not hold. The analysis is as follows: 


$$
m_{D}=\min _{i}\left\{d_{i}+r_{d a}\right\}=\min _{i}\left\{0, d_{i}+r_{d a}\right\} .
$$

It is obvious that the value of the TCD is not less than 0 . If the DM selection preference is applied, the minimum value of the target centre is 0 , i.e., $m_{D}=0$.

$$
\begin{gathered}
M_{D}-m_{D}=\max _{i}\left\{d_{i}+r_{d a}\right\}-\min _{i}\left\{d_{i}+r_{d a}\right\} \\
=\max _{i}\left\{d_{i}+r_{d a}\right\}-\min _{i}\left\{0, d_{i}+r_{d a}\right\} \\
=\max _{i}\left\{d_{i}+r_{d a}\right\}-0 \\
=\max _{i}\left\{d_{i}\right\}-\left|r_{d a}\right|, \\
M_{d}-m_{d}=\max _{i}\left\{d_{i}\right\}-\min _{i}\left\{d_{i}\right\} . \\
\text { Obviously, }\left|r_{d a}\right|>\min _{i}\left\{d_{i}\right\} . \text { Thus, } \\
M_{D}-m_{D}<M_{d}-m_{d} .
\end{gathered}
$$

Comparing equations (18) and (21), $\Delta Z_{d}>\Delta Z_{a}$, which means that the target centre decided by DM selection preference can enlarge the difference of the index TCDs. Importantly, however, these index TCDs have no difference (all can be seen as having values of 0 ) when their index values are superior to the target centre index value. This indicates that the target centre determined by DM selection preferences has the function of "rewarding good and punishing bad."

3.2.2. Vector Normalisation Method. The normalised difference of the two TCDs with the vector normalisation method regarding a different target centre is obtained using equations (25) and (26) according to the above situation:

$$
\begin{gathered}
\Delta Z_{a}=\frac{d_{i_{0}+1}}{\sqrt{\sum_{i=1}^{n} d_{i}^{2}}}-\frac{d_{i_{0}}}{\sqrt{\sum_{i=1}^{n} d_{i}^{2}}}=\frac{d_{i_{0}+1}-d_{i_{0}}}{\sqrt{\sum_{i=1}^{n} d_{i}^{2}}} \\
\Delta Z_{d}=\frac{D_{i_{0}+1}}{\sqrt{\sum_{i=1}^{n} D_{i}^{2}}}-\frac{D_{i_{0}}}{\sqrt{\sum_{i=1}^{n} D_{i}^{2}}}=\frac{D_{i_{0}+1}-D_{i_{0}}}{\sqrt{\sum_{i=1}^{n} D_{i}^{2}}}
\end{gathered}
$$

Equation (26) can also be rewritten as (27) via (18):

$$
\Delta Z_{d}=\frac{\left(d_{i_{0}+1}+r_{d a}\right)-\left(d_{i_{0}}+r_{d a}\right)}{\sqrt{\sum_{i=1}^{n}\left(d_{i}+r_{d a}\right)^{2}}}=\frac{d_{i_{0}+1}-d_{i_{0}}}{\sqrt{\sum_{i=1}^{n}\left(d_{i}+r_{d a}\right)^{2}}} .
$$

Comparing equations (25) and (27), the following can be concluded.

(1) If $r_{d a}>0$, then $\Delta Z_{d}<\Delta Z_{a}$, which means that the target centre is decided by DM desires and can reduce the difference of the index TCDs.

(2) If $r_{d a}<0$, then $\Delta Z_{d}>\Delta Z_{a}$, which means that the target centre is decided by DM selection preferences and can magnify the difference of index TCDs. It should be noted, however, that these index TCDs have no difference (all can be regarded as having values of 0 ) when their index values are superior to the target centre index value. This proves that the target centre determined by DM selection preferences has the function of "rewarding good and punishing bad."

3.2.3. Linear Proportional Normalisation Method. The difference of the two normalised TCDs with the linear proportional normalisation method regarding a different target centre is obtained using equations (28) and (29) according to the above situation:

$$
\begin{gathered}
\Delta Z_{a}=\frac{d_{i_{0}+1}}{d_{M}}-\frac{d_{i_{0}}}{d_{M}}=\frac{d_{i_{0}+1}-d_{i_{0}}}{d_{M}}, \\
\Delta Z_{d}=\frac{D_{i_{0}+1}}{D_{M}}-\frac{D_{i_{0}}}{D_{M}}=\frac{D_{i_{0}+1}-D_{i_{0}}}{D_{M}} .
\end{gathered}
$$

Because $d_{M}=\max _{i}\left\{d_{i}\right\}$ and $D_{M}=\max _{i}\left\{D_{i}\right\}$, Equation (29) can be rewritten as

$$
\Delta Z_{d}=\frac{\left(d_{i_{0}+1}+r_{d a}\right)-\left(d_{i_{0}}+r_{d a}\right)}{d_{M}+r_{d a}}=\frac{d_{i_{0}+1}-d_{i_{0}}}{d_{M}+r_{d a}} .
$$

Comparing equations (28) and (30), the following can be concluded.

(1) If $r_{d a}>0$, then $\Delta Z_{d}<\Delta Z_{a}$, which means that the target centre is decided by DM desires and can decrease the difference of index TCDs.

(2) If $r_{d a}<0$, then $\Delta Z_{d}>\Delta Z_{a}$, which means that the target centre is decided by DM selection preferences and can increase the difference of index TCDs. It should be noted, however, that these index TCDs are the same when their index values are superior to the target centre index value. This means that the target centre determined by DM selection preferences has the function of "rewarding good and punishing bad."

3.2.4. Analysis of Each Normalisation Method. Different normalisation methods affect index TCDs in different ways regarding a target centre determined by DM preferences. In brief, normalisation methods can be classified into two types with regard to their impact on the index TCDs owing to a variable target centre: those that affect index TCDs, and those that do not. The min-max normalisation method does not impact index TCDs when the target centre is determined by DM desires. Others methods do impact index TCDs, including the min-max normalisation method when it is used with DM selection preferences, as well as the vector normalisation method, the linear proportional normalisation method, and the linear normalisation method. The min-max normalisation method with DM desires does not impact index TCDs because it internally removes those factors that affect the normalization of index TCDS. By contrast, index TCDs are affected by the min-max normalisation method with DM selection preferences-and other normalisation methods-because some of these factors are not removed. 
Therefore, there are important differences among normalisation methods with regard to their impact on index TCDs, and this can be explained be the different normalisation mechanisms. However, the extent of this impact requires further research.

\subsection{Effect of Different Normalisation Methods on Objective} Weights. The impact of different normalisation methods on index TCDs has thus been discussed. Furthermore, the effect of these methods on objective weights can be analysed with the proposed method because these weights are mainly determined based on changes to the normalised TCDs. The following analysis pertains to different normalisation methods and their effect on objective weights.

From equations (7)-(9), objective weights are affected by normalised TCDs. As discussed above, normalisation methods can be classified into two types with regard to their effect on index TCDs considering different kinds of DM preferences. Accordingly, the effect that these methods have on objective weights can also be divided into two types.

3.3.1. Objective Weights Obtained by the Min-Max Normalisation Method. We observed above that TCDs with a target centre determined by DM desires are not affected by the min-max normalisation method. As such, the objective weights obtained will likewise remain unaffected when the target centre is determined by DM desires. However, they will be affected when the target centre is determined the DM selection preferences with the min-max normalisation method.

3.3.2. Objective Weights Obtained by Other Normalisation Methods. Except for the min-max normalisation method, other normalisation methods will affect normalised TCDs when the target indices are determined by DM preferences. As such, their objective weights will be affected as well. Furthermore, different normalisation methods with different principles will impact these objective weights to different degrees.

3.4. Numerical Example. To verify the above conclusions, a numerical example is provided. Suppose that $S_{i c}=(1.8,1.4$, $2.7,1.2,3.6,2.4,1.3,2.1,1.5,1.7)$ is a set of alternative indices under attribute $A_{C}$, and these values are benefit-type indices. The following analysis is based on target centre indices determined by the alternatives themselves, DM desires, and DM selection preferences with different normalisation methods.

3.4.1. Target Centre Index and TCD. The target centre index is calculated to be 3.6 using equation (10), and this originates from the alternatives themselves. Also, 4.0 and 2.0 represent target centre indices decided by DM desires and DM selection preferences, respectively. Then, the TCDs can be calculated using equation (17). Let $R_{a}=(1.8,1.4,2.7,1.2,3.6$,
2.4 1.3, 2.1, 1.5, 1.7), $R_{d d}=(1.8,1.4,2.7,1.2,3.6,2.4,1.3,2.1$, $1.5,1.7)$, and $R_{d s}=(1.8,1.4,2.7,1.2,3.6,2.4,1.3,2.1,1.5,1.7)$ be the TCD vectors with the target centre indices determined by the alternatives themselves, DM desires, and DM selection preferences, respectively.

3.4.2. Normalisation by Different Methods. Let $r_{a}, r_{d s}$, and $r_{d s}$ denote normalised TCDs with a target centre determined by alternatives, DM desires, and DM's selection preferences, respectively. Table 1 lists the results of TCDs normalised by the four different methods.

As seen in Table 1, different normalisation methods affect the TCDs to different degrees. The result of the minmax method with the target centre index determined by DM desires is identical to that of the target centre index determined by alternatives, although it differs from a target centre index determined by DM selection preferences. The results of other normalisation methods with different target centre indices are all different. The numerical example thus confirms the above analysis.

3.4.3. Analysis of the Effect of Normalisation Methods on Objective Weights. Table 2 lists the sums of deviations of TCDs normalised by different methods. Figure 2 illustrates a comparison of these sums of deviations. In Table 2, $z_{a}, z_{d d}$, and $z_{d s}$ denote the sums of deviations of TCDs with a target centre determined by the alternatives, DM desires, and DM selection preferences, respectively. From Table 2 and Figure 2, we arrive at the following conclusions.

(1) Whether or not DM preferences are considered with regard to the target centre, the sums of deviations of normalised TCDs differ depending on the normalisation method. Consequently, the objective weights also differ.

(2) There is no difference in the sums of deviations of normalised TCDs with the target centre determined by alternatives and DM desires with the min-max normalisation method. However, the sums of deviations with other normalisation methods are indeed different, which means that the objective weights are different as well.

(3) Except for the min-max method, the sums of deviations of normalised TCDs processed by other normalisation methods have the following characteristics. Among the sums of deviations of normalised TCDs, the lowest sum of deviations originates from a target centre determined by DM desires. By contrast, the highest sum of deviations originates from a target centre determined by DM selection preferences.

3.5. Discussion and Suggestions. In general, our results indicate that different normalisation methods affect index TCDs in different ways considering target centre indices determined by DM preferences. In brief, normalisation methods can be classified into two types based on their effect 
TABle 1: Target centre distances normalised by different methods.

\begin{tabular}{|c|c|c|c|c|c|c|c|c|c|c|c|}
\hline$S_{i c}$ & & 1.8 & 1.4 & 2.7 & 1.2 & 3.6 & 2.4 & 1.3 & 2.1 & 1.5 & 1.7 \\
\hline$R_{a} / 3.6$ & & 1.8 & 2.2 & 0.9 & 2.4 & 0 & 1.2 & 2.3 & 1.5 & 2.1 & 1.9 \\
\hline$R_{d d} / 4.0$ & & 2.2 & 2.6 & 1.3 & 2.8 & 0.4 & 1.6 & 2.7 & 1.9 & 2.5 & 2.3 \\
\hline$R_{d s} / 2.0$ & & 0.2 & 0.6 & 0 & 0.8 & 0 & 0 & 0.7 & 0 & 0.5 & 0.3 \\
\hline \multirow{3}{*}{ Min-max method } & $r_{a}$ & 0.7500 & 0.9167 & 0.3750 & 1.0000 & 0 & 0.5000 & 0.9583 & 0.6250 & 0.8750 & 0.7917 \\
\hline & $r_{d s}$ & 0.7500 & 0.9167 & 0.3750 & 1.0000 & 0 & 0.5000 & 0.9583 & 0.6250 & 0.8750 & 0.7917 \\
\hline & $r_{d s}$ & 0.2500 & 0.7500 & 0 & 1.0000 & 0 & 0 & 0.8750 & 0 & 0.6250 & 0.3750 \\
\hline \multirow{3}{*}{ Vector method } & $r_{a}$ & 0.3200 & 0.3911 & 0.1600 & 0.4266 & 0 & 0.2133 & 0.4088 & 0.2666 & 0.3733 & 0.3377 \\
\hline & $r_{d d}$ & 0.3234 & 0.3821 & 0.1911 & 0.4115 & 0.0588 & 0.2352 & 0.3968 & 0.2793 & 0.3674 & 0.3381 \\
\hline & $r_{d s}$ & 0.1463 & 0.4388 & 0 & 0.5850 & 0 & 0 & 0.5119 & 0 & 0.3656 & 0.2194 \\
\hline \multirow{3}{*}{ Linear proportional method } & $r_{a}$ & 0.7500 & 0.9167 & 0.3750 & 1.0000 & 0 & 0.5000 & 0.9583 & 0.6250 & 0.8750 & 0.7917 \\
\hline & $r_{d d}$ & 0.7857 & 0.9286 & 0.4643 & 1.0000 & 0.1429 & 0.5714 & 0.9643 & 0.6786 & 0.8929 & 0.8214 \\
\hline & $r_{d s}$ & 0.2500 & 0.7500 & 0 & 1.0000 & 0 & 0 & 0.8750 & 0 & 0.6250 & 0.3750 \\
\hline \multirow{3}{*}{ Linear method } & $r_{a}$ & 0.1104 & 0.1350 & 0.0552 & 0.1472 & 0 & 0.0736 & 0.1411 & 0.0920 & 0.1288 & 0.1166 \\
\hline & $r_{d d}$ & 0.1084 & 0.1281 & 0.0640 & 0.1379 & 0.0197 & 0.0788 & 0.1330 & 0.0936 & 0.1232 & 0.1133 \\
\hline & $r_{d s}$ & 0.0645 & 0.1935 & 0 & 0.2581 & 0 & 0 & 0.2258 & 0 & 0.1613 & 0.0968 \\
\hline
\end{tabular}

TABLE 2: Sums of deviations of TCDs normalised by different methods.

\begin{tabular}{lcccc}
\hline & Min-max method & Vector method & Linear proportional method & Linear method \\
\hline$z_{a}$ & 2.2917 & 0.9776 & 2.2917 & 0.3374 \\
$z_{d d}$ & 2.2917 & 0.8084 & 1.9643 & 0.2709 \\
$z_{d s}$ & 3.3750 & 1.9744 & 3.3750 & 0.8710 \\
\hline
\end{tabular}

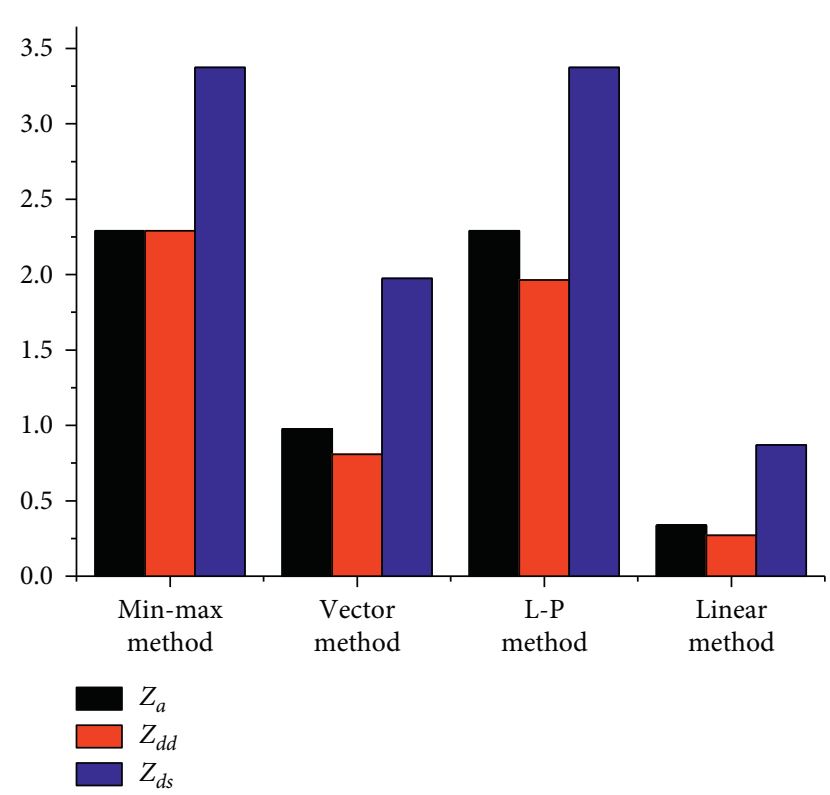

FIgURE 2: Comparison of sums of deviations of normalised TCDs.

on index TCDs: those methods that affect the index TCDs, and those that do not. Specifically, the min-max normalisation method does not affect index TCDs when the target centre is determined by DM desires. However, the min-max method does affect index TCDs when the target centre is determined by DM selection preferences-as do other normalisation methods. Based on this, the corresponding impact of different normalisation methods on objective weights can also be determined. In this work, only one objective method was studied. Indeed, the weights are determined according to the changes of TCDs, which are, in turn, affected by different normalisation methods. Our results show that all normalisation methods impact the objective weights, with the exception of the min-max normalisation method when the target centre is determined DM desires.

\section{Applications}

4.1. Data. The data in [19] were used to exemplify our results. We evaluated the comprehensive safety of ten coal mines with eight indices: seam $\operatorname{dip}\left({ }^{\circ}\right)$, methane emission rate $\left(\mathrm{m}^{3} / \mathrm{t}\right)$, water inflow $\left(\mathrm{m}^{3} / \mathrm{h}\right)$, spontaneous combustion period (months), ventilating structures qualified rate (\%), equivalent orifice, mortality per million tons (person/10 $\mathrm{t}$ ), and accident-related economic losses $\left(10^{5} \mathrm{CNY}\right)$, denoted by $A_{1}$ through $A_{8}$, respectively, with the alternatives denoted by $S_{1}$ through $S_{10}$. The data are shown in Table 3. Here, $A_{4}$ to $A_{6}$ are benefit-type attributes, and the others are cost-type attributes. The DM attribute preferences are $A_{2}, A_{5}, A_{6}$, and $A_{7}$ with values of $0,95,2.0$, and 0.2 , respectively [19].

\subsection{Calculations}

4.2.1. Obtaining Final Target Centre. The target centre vector $C_{a}=(10,3.7,120,12,100,3.6,0,300)$ is obtained using equation (10). Given DM preferences, the final target centre vector can be revised to $C_{d}=(10,0,120,12,95,2.0,0.2,300)$ using equation (14).

4.2.2. Calculating Original TCDs. All index TCDs can be calculated from the target centre decided by DM preferences, as shown in Table 4. 
TABLe 3: Safety data for coal mines.

\begin{tabular}{lcccccccc}
\hline$S_{i}$ & $A_{1}$ & $A_{2}$ & $A_{3}$ & $A_{4}$ & $A_{5}$ & $A_{6}$ & $A_{7}$ \\
\hline$S_{1}$ & 21 & 6 & 220 & 12 & 92 & 1.8 & 0.18 \\
$S_{2}$ & 16 & 3.7 & 200 & 6 & 90 & 1.4 & 0.712 & 581 \\
$S_{3}$ & 26 & 9.2 & 180 & 10 & 88 & 2.7 & 1.34 \\
$S_{4}$ & 10 & 4 & 260 & 8 & 94 & 1.2 & 0 & 1051.6 \\
$S_{5}$ & 30 & 8.2 & 350 & 10 & 96 & 3.6 & 442.5 \\
$S_{6}$ & 19 & 5 & 130 & 12 & 100 & 2.4 & 0.641 & 0 \\
$S_{7}$ & 17 & 9.6 & 400 & 6 & 86 & 1.3 & 300 \\
$S_{8}$ & 40 & 14 & 600 & 6 & 95 & 2.1 & 1.23 \\
$S_{9}$ & 12 & 12.8 & 120 & 10 & 91 & 1.5 & 0.872 \\
$S_{10}$ & 14 & 5.8 & 155 & 12 & 89 & 1.7 & 864.7 \\
\hline
\end{tabular}

TABLE 4: All index target centre distances.

\begin{tabular}{|c|c|c|c|c|c|c|c|c|}
\hline$r_{i j}$ & $A_{1}$ & $A_{2}$ & $A_{3}$ & $A_{4}$ & $A_{5}$ & $A_{6}$ & $A_{7}$ & $A_{8}$ \\
\hline$\overline{r_{1 j}}$ & 11 & 6 & 100 & 0 & 3 & 0.2 & 0 & 81 \\
\hline$r_{2 j}$ & 6 & 3.7 & 80 & 6 & 5 & 0.6 & 0.512 & 264 \\
\hline$r_{3 j}$ & 16 & 9.2 & 60 & 2 & 7 & 0 & 1.14 & 751.6 \\
\hline$r_{4 j}$ & 0 & 4 & 140 & 4 & 1 & 0.8 & 0 & 142.5 \\
\hline$r_{5 j}$ & 20 & 8.2 & 230 & 2 & 0 & 0 & 0.441 & 488 \\
\hline$r_{6 j}$ & 9 & 5 & 10 & 0 & 0 & 0 & 0 & 0 \\
\hline$r_{7 j}$ & 7 & 9.6 & 280 & 6 & 9 & 0.7 & 1.03 & 664.7 \\
\hline$r_{8 j}$ & 30 & 14 & 480 & 6 & 0 & 0 & 0.92 & 585.6 \\
\hline$r_{9 j}$ & 2 & 12.8 & 0 & 2 & 4 & 0.5 & 0.672 & 539.3 \\
\hline$r_{10 j}$ & 4 & 5.8 & 35 & 0 & 6 & 0.3 & 0.226 & 317.2 \\
\hline
\end{tabular}

TABLE 5: Different objective weights with DM preferences.

\begin{tabular}{lcccccccc}
\hline$W_{j}$ & $A_{1}$ & $A_{2}$ & $A_{3}$ & $A_{4}$ & $A_{5}$ & $A_{6}$ & $A_{7}$ \\
\hline$W_{1}$ & 0.0741 & 0.0935 & 0.0685 & 0.2449 & 0.1512 & 0.1281 & 0.1410 & 0.0985 \\
$W_{2}$ & 0.1103 & 0.0420 & 0.1209 & 0.1998 & 0.1739 & 0.1351 & 0.1356 \\
$W_{3}$ & 0.0774 & 0.0529 & 0.0716 & 0.2559 & 0.1580 & 0.1339 & 0.1474 & 0.0826 \\
$W_{4}$ & 0.1068 & 0.0286 & 0.1393 & 0.1986 & 0.1766 & 0.1507 & 0.1326 & 0.0669 \\
\hline
\end{tabular}

4.2.3. Obtaining Objective Weights. Our study demonstrates that different normalisation methods can affect objective weights owing to the variation in normalised index values. Table 5 lists the objective weights calculated by different normalisation methods using equations (7)-(9).

In Table $5, W_{1}, W_{2}, W_{3}$, and $W_{4}$ represent weight vectors affected by the min-max normalisation method, vector normalisation method, linear proportional normalisation method, and linear normalisation method, respectively.

Figure 3 compares different types of objective weights. As seen in Figure 3, each type of objective weights differs from the other. This is because the different normalisation methods affect these objective weights.

4.2.4. Decision-Making with Integrated TCDs. Having obtained the objective weights, the integrated normalised TCDs can be calculated. The results are given in Table 6 .

As seen in Table 6, the ranking of the alternatives with different objective weights remains the same, although the values of integrated TCDs are different. This result shows that different normalisation methods might not impact the ultimate result of the ranked alternatives, even though the objective weights are affected by them. It should be noted

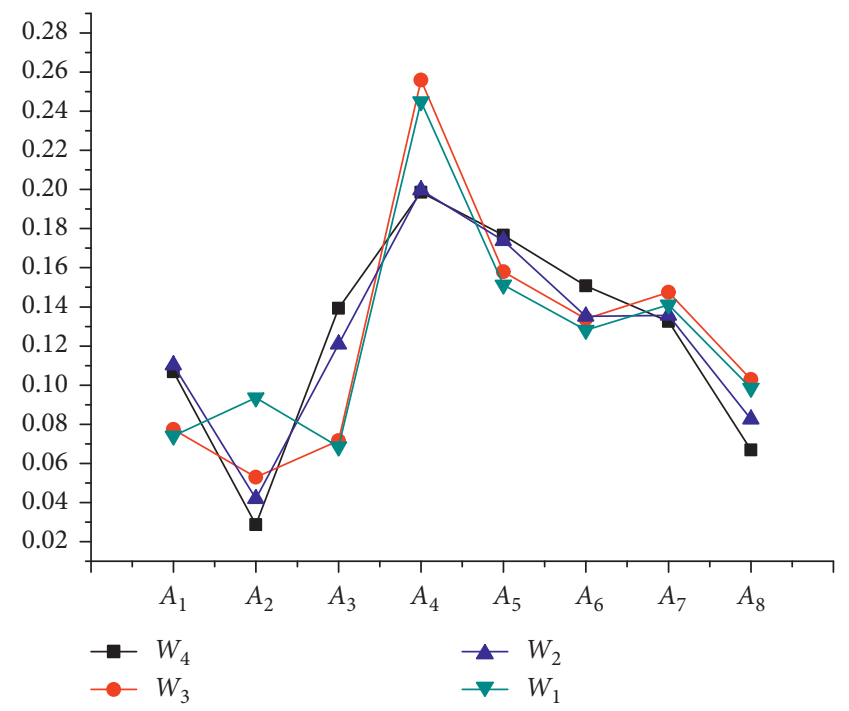

FIgURE 3: Comparison of different types of objective weights with DM preferences.

that the specific ranking of the alternatives with the GTDM remains uncertain when the target centre indices are partially determined by DM preferences depending on the 
TABLE 6: Ranked alternatives from different normalisation methods with DM preferences.

\begin{tabular}{cccccccccc}
\hline & \multicolumn{3}{c}{$\begin{array}{c}\text { Min-max } \\
\text { method }\end{array}$} & \multicolumn{2}{c}{$\begin{array}{c}\text { Vector } \\
\text { method }\end{array}$} & \multicolumn{2}{c}{$\begin{array}{c}\text { Linear } \\
\text { proportional } \\
\text { method }\end{array}$} & \multicolumn{2}{c}{$\begin{array}{c}\text { Linear } \\
\text { method }\end{array}$} \\
& \multicolumn{2}{c}{$r$} & Rank & $r$ & Rank & $r$ & Rank & $r$ & Rank \\
\hline$S_{1}$ & 0.1554 & 2 & 0.1163 & 2 & 0.1632 & 2 & 0.0495 & 2 \\
$S_{2}$ & 0.5492 & 8 & 0.3065 & 8 & 0.5878 & 8 & 0.1306 & 8 \\
$S_{3}$ & 0.5368 & 7 & 0.3023 & 7 & 0.5435 & 7 & 0.1187 & 7 \\
$S_{4}$ & 0.3496 & 5 & 0.2004 & 5 & 0.3775 & 5 & 0.0900 & 5 \\
$S_{5}$ & 0.3233 & 4 & 0.1992 & 4 & 0.3261 & 4 & 0.0805 & 4 \\
$S_{6}$ & 0.0355 & 1 & 0.0327 & 1 & 0.0436 & 1 & 0.0120 & 1 \\
$S_{7}$ & 0.8337 & 10 & 0.4708 & 10 & 0.8513 & 10 & 0.1994 & 10 \\
$S_{8}$ & 0.6717 & 9 & 0.3873 & 9 & 0.6570 & 9 & 0.1603 & 9 \\
$S_{9}$ & 0.4703 & 6 & 0.2314 & 6 & 0.4534 & 6 & 0.0928 & 6 \\
$S_{10}$ & 0.2523 & 3 & 0.1596 & 3 & 0.2656 & 3 & 0.0661 & 3 \\
\hline
\end{tabular}

TABle 7: Different objective weights without DM preferences.

\begin{tabular}{ccccccccc}
\hline$W_{j}$ & $A_{1}$ & $A_{2}$ & $A_{3}$ & $A_{4}$ & $A_{5}$ & $A_{6}$ & $A_{7}$ & $A_{8}$ \\
\hline$W_{1}$ & 0.0883 & 0.1114 & 0.0685 & 0.2918 & 0.1040 & 0.0704 & 0.1483 & 0.1174 \\
$W_{2}$ & 0.1357 & 0.1316 & 0.1305 & 0.2458 & 0.0828 & 0.0407 & 0.1312 & 0.1016 \\
$W_{3}$ & 0.0883 & 0.1114 & 0.0685 & 0.2918 & 0.1040 & 0.0704 & 0.1483 & 0.1174 \\
$W_{4}$ & 0.1380 & 0.1327 & 0.1747 & 0.2566 & 0.0625 & 0.0292 & 0.1199 & 0.0864 \\
\hline
\end{tabular}

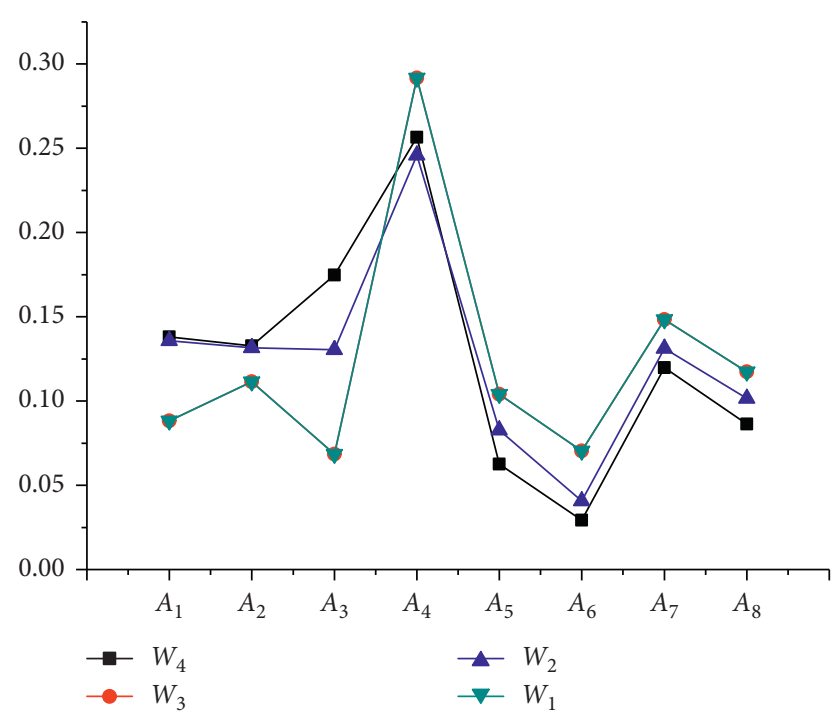

FIgURE 4: Comparison of different types of objective weights without DM preferences.

normalisation method. The following can thus be inferred. First, the above analysis of the effect of normalisation methods on alternatives focuses on the index TCD. However, ultimate decision-making is based on an integrated TCD, which reflects the aggregation of all index TCDs of an alternative. Second, the variable target centre indices determined by DM preferences affect TCDs in different ways. They can improve the ranking of alternatives or worsen it. These two opposing trends that affect index TCDs render the ranking of alternatives uncertain. Third, different weights can also affect the results of decision-making in opposing directions.
TABLE 8: Ranked alternatives from different normalisation methods without DM preferences.

\begin{tabular}{cccccccccc}
\hline & \multicolumn{3}{c}{$\begin{array}{c}\text { Min-max } \\
\text { method }\end{array}$} & \multicolumn{2}{c}{$\begin{array}{c}\text { Vector } \\
\text { method }\end{array}$} & \multicolumn{3}{c}{$\begin{array}{c}\text { Linear } \\
\text { proportional } \\
\text { method }\end{array}$} & \multicolumn{2}{c}{$\begin{array}{c}\text { Linear } \\
\text { method }\end{array}$} \\
& \multicolumn{1}{c}{ Rank } & $r$ & Rank & $r$ & Rank & $r$ & Rank \\
\hline$S_{1}$ & 0.2163 & 2 & 0.1253 & 2 & 0.2163 & 2 & 0.0498 & 3 \\
$S_{2}$ & 0.5797 & 7 & 0.2631 & 6 & 0.5797 & 7 & 0.1044 & 6 \\
$S_{3}$ & 0.5936 & 8 & 0.3122 & 8 & 0.5936 & 8 & 0.1177 & 8 \\
$S_{4}$ & 0.3407 & 4 & 0.1402 & 4 & 0.3407 & 4 & 0.0552 & 4 \\
$S_{5}$ & 0.4145 & 5 & 0.2678 & 7 & 0.4145 & 5 & 0.1156 & 7 \\
$S_{6}$ & 0.0772 & 1 & 0.0492 & 1 & 0.0772 & 1 & 0.0195 & 1 \\
$S_{7}$ & 0.8275 & 9 & 0.4218 & 9 & 0.8275 & 9 & 0.1731 & 9 \\
$S_{8}$ & 0.8565 & 10 & 0.5268 & 10 & 0.8565 & 10 & 0.2317 & 10 \\
$S_{9}$ & 0.5574 & 6 & 0.2442 & 5 & 0.5574 & 6 & 0.0893 & 5 \\
$S_{10}$ & 0.2736 & 3 & 0.1270 & 3 & 0.2736 & 3 & 0.0437 & 2 \\
\hline
\end{tabular}

\subsection{Comparison and Analysis}

4.3.1. Decision-Making without considering DM Preferences. We used target centre indices determined by alternatives to draw a comparison with target centre indices partially decided by DM preferences based on the data shown in Table 3. The objective weights were calculated with TCDs normalised by different methods. The objective weights are shown in Table 7 and Figure 4.

As seen in Table 7, the objective weights from different normalisation methods are different from those shown in Table 5. In Table 7, however, objective weights obtained with the min-max normalisation method are identical to those obtained with the linear proportional normalisation method. In Figure 4, the curves $W_{1}$ and $W_{3}$ overlap, which means that they have exactly the same weight. The reason for this is that the TCDs normalised by these two methods are the same. However, this is not always the true for the linear proportional normalisation method when different parameters are set (e.g., $M_{j}$ can be set to a different value in equation (5)).

Table 8 shows the ranking of alternatives with target centres determined by alternatives and normalised by different methods. The results in Table 8 are conspicuously different from those in Table 6 .

4.3.2. Results Analysis. With different objective weights, the integrated TCDs are different. However, the ranking of the alternatives remains the same: $S_{6}>S_{1}>S_{10}>S_{5}>S_{4}>S_{9}>S_{3}>S_{2}>$ $S_{8}>S_{7}$. Furthermore, the integrated TCDs also differ without considering the target centre indices determined by DM preferences. As such, the results do not remain the same; there are two ranking orders. The results from min-max normalisation and linear proportional normalisation are identical, with the following ranking: $S_{6}>S_{1}>S_{4}>S_{10}>S_{5}>$ $S_{2}>S_{9}>S_{3}>S_{8}>S_{7}$. The results with vector normalisation and linear normalisation are almost the same, but for a slight change with $S_{1}$ and $S_{10}$. The results obtained by the proposed method, however, retain the same order of ranking, even with different normalisation methods. However, the ranking of the alternatives usually differs when a target centre is 
determined by the alternatives themselves. Overall, the ranking is consistent when the target centre is partially determined by DM preferences, despite the type of normalisation method.

\section{Conclusions}

This work arrived at the following conclusions.

(1) Different normalisation methods can impact index TCDs when the target centre index is determined by DM preferences. Among the four normalisation methods we tested, the min-max normalisation method was the only one that did not impact index TCDs, and only when the target centre was determined by DM desires. When DM selection preferences were used to determine the target centre, the min-max normalisation method did affect the index TCDs, as did the other normalisation methods we tested.

(2) Objective weights are derived from the index TCDs. As such, different normalisation methods indeed affect the objective weights.

(3) Although the choice of normalisation method impacts the index TCDs, the ranking of alternatives is little affected or completely unaffected by this choice because these rankings are based on integrated TCDs.

Importantly, the proposed method considered only a few commonly used normalisation methods. Furthermore, the extent of the effect of different normalisation methods on alternatives and weights was not addressed. As such, in future research, we will further investigate the mechanisms of normalisation methods and their effect on the ranking of alternatives.

\section{Data Availability}

The data used in this study were originated from Reference 19.

\section{Conflicts of Interest}

The authors declare that there are no conflicts of interest regarding the publication of this article.

\section{Acknowledgments}

The authors acknowledge support from a Doctoral Fund at Henan Polytechnic University (grant no. B2016-53) and Fundamental Research Funds for Henan Province Universities (grant no. SKJZD2019-05).

\section{References}

[1] J. L. Deng, Grey System Theory, Huangzhong University of Science and Technology Press, Wuhan, China, 2002.

[2] Y. G. Dang, "Multi-attribute decision model of grey target considering weights," Statistics and Decision, no. 3, pp. 29-30, 2004.
[3] S. Liu, W. Yuan, and K. Sheng, "Multi-attribute intelligent grey target decision method," Control and Decision, vol. 25, no. 8, pp. 1159-1163, 2010.

[4] J. Zhu and K. W. Hipel, "Multiple stages grey target decision making method with incomplete weight based on multigranularity linguistic label," Information Sciences, vol. 212, pp. 15-32, 2012.

[5] C. G. Shen, Y. G. Dang, and L. L. Pei, "Hybrid multi-attribute decision model of grey target," Statistics and Decision, no. 12, pp. 17-20, 2010.

[6] D. Luo and X. Wang, "The multi-attribute grey target decision method for attribute value within three-parameter interval grey number," Applied Mathematical Modelling, vol. 36, no. 5, pp. 1957-1963, 2012.

[7] J. Song and Y.-G. Dang, "The decision-making model of harden grey target based on interval number with preference information on alternatives," Journal of Grey System, vol. 21, no. 3, pp. 291-300, 2009.

[8] J. Ma and C. Ji, "Generalized grey target decision method for mixed attributes based on connection number," Journal of Applied Mathematics, vol. 2014, Article ID 763543, 8 pages, 2014.

[9] H. H. Wang, J. Zhu, and Z.-G. Fang, "Grey target cluster decision method on linguistic evaluation case-based," Systems Engineering-Theory \& Practice, vol. 33, no. 12, pp. 31723181, 2013.

[10] W. Qian, X. Yang, and J. Li, "Grey target decision model based on interval grey number type panel data and its application," Journal of Grey System, vol. 30, no. 1, pp. 69-80, 2018.

[11] J. Ma, "Generalised grey target decision method for mixed attributes with index weights containing uncertain numbers," Journal of Intelligent \& Fuzzy Systems, vol. 34, no. 1, pp. 625-632, 2018.

[12] J. Song, Y.-G. Dang, X.-M. Li, and Z.-X. Wang, "Grey group decision-making model discriminable for invalid decision values," System Engineering and Electronics, vol. 33, no. 6, pp. 1317-1320, 2011.

[13] W. Dai and J. Li, "Off-target deviation degree method for grey multi-attribute group decision-making," Systems Engineering-Theory \& Practice, vol. 34, no. 3, pp. 787-792, 2014.

[14] Y. Liu, "Multi-objective grey target decision-making based on prospect theory," Control and Decision, vol. 28, no. 3, pp. 345-350, 2013.

[15] J. Zhu, L.-L. Zhang, Y.-H. Liang, and L. Peng, "Grey target decision method based on uncertain evidence aggregation under conflict interest participants," Control and Decision, vol. 27, no. 7, pp. 1037-1041, 2012.

[16] B. Zeng, C. Li, and S.-F. Liu, "A novel grey target decisionmaking model based on cobweb area and its application for choosing the software development pattern," Scientia Iranica, vol. 23, no. 1, pp. 361-373, 2016.

[17] M. Hu, "Grey target decision model based on a new distance measure," Journal of Grey System, vol. 28, no. 2, pp. 27-34, 2016.

[18] P. Li, L. Jian, S. Liu, and X. Su, "Grey target method for intuitionistic fuzzy decision making based on grey incidence analysis," Journal of Grey System, vol. 28, no. 4, pp. 96-109, 2016.

[19] J. Ma, "Grey target decision method for a variable target centre based on the decision maker's preferences," Journal of Applied Mathematics, vol. 2014, Article ID 572529, 6 pages, 2014.

[20] J. Ma, "Generalized grey target decision method for mixed attributes based on kullback-leibler distance," Entropy, vol. 20, no. 7, p. 523, 2018. 
[21] J. Ma, "Generalised grey target decision method for mixed attributes based on the improved Gini-Simpson index," Soft Computing, vol. 23, no. 24, pp. 13449-13458, 2019.

[22] J. Ma, "Gini-Simpson index based generalised grey target decision method for mixed attributes," Journal of Intelligent \& Fuzzy Systems, vol. 37, no. 2, pp. 2597-2608, 2019.

[23] J. Ma and J. Sun, "Grey target decision method for positive and negative target centers based on decision maker's preferences," Science and Technology Management Research, vol. 35, no. 23, pp. 185-190, 2014.

[24] L. Li, Y. Guo, and P. Yi, "Analyzing the principles for choosing dimensionless methods," Journal of Systems \& Management, vol. 25, no. 6, pp. 1040-1045, 2016.

[25] M. Zhan, Z. Liao, and J. Xu, "Character analysis of linear methods," Statistics \& Information Forum, vol. 31, no. 12, pp. 17-22, 2016.

[26] X. Liu, "Research on the index non-liner standardization based on S type curve," Statistics \& Information Forum, vol. 33, no. 2, pp. 17-21, 2018.

[27] A. Jahan and K. L. Edwards, "A state-of-the-art survey on the influence of normalization techniques in ranking: improving the materials selection process in engineering design," $M a-$ terials \& Design, vol. 65, pp. 335-342, 2015.

[28] R. F. d. F. Aires and L. Ferreira, "A new approach to avoid rank reversal cases in the TOPSIS method," Computers \& Industrial Engineering, vol. 132, pp. 84-97, 2019.

[29] P. Chen, "Effects of normalization on the entropy-based TOPSIS method," Expert Systems with Applications, vol. 136, pp. 33-41, 2019.

[30] M. Behzadian, S. Khanmohammadi Otaghsara, M. Yazdani, and J. Ignatius, "A state-of the-art survey of TOPSIS applications," Expert Systems with Applications, vol. 39, no. 17, pp. 13051-13069, 2012.

[31] J. Ren, "Technology selection for ballast water treatment by multi-stakeholders: a multi-attribute decision analysis approach based on the combined weights and extension theory," Chemosphere, vol. 191, pp. 747-760, 2018.

[32] L. Chen and J. Ren, "Multi-attribute sustainability evaluation of alternative aviation fuels based on fuzzy ANP and fuzzy grey relational analysis," Journal of Air Transport Management, vol. 68, pp. 176-186, 2018.

[33] S. Jain, S. Shukla, and R. Wadhvani, "Dynamic selection of normalization techniques using data complexity measures," Expert Systems with Applications, vol. 106, pp. 252-262, 2018.

[34] Y. Guo, Theory, Method and Application of Comprehensive Evaluation, Science Press, Beijing, China, 2007. 


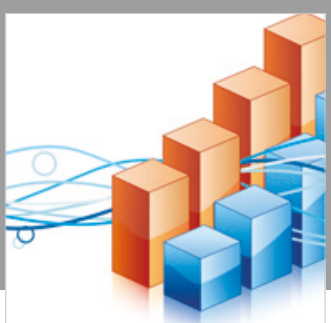

Advances in

Operations Research

\section{-n-m}
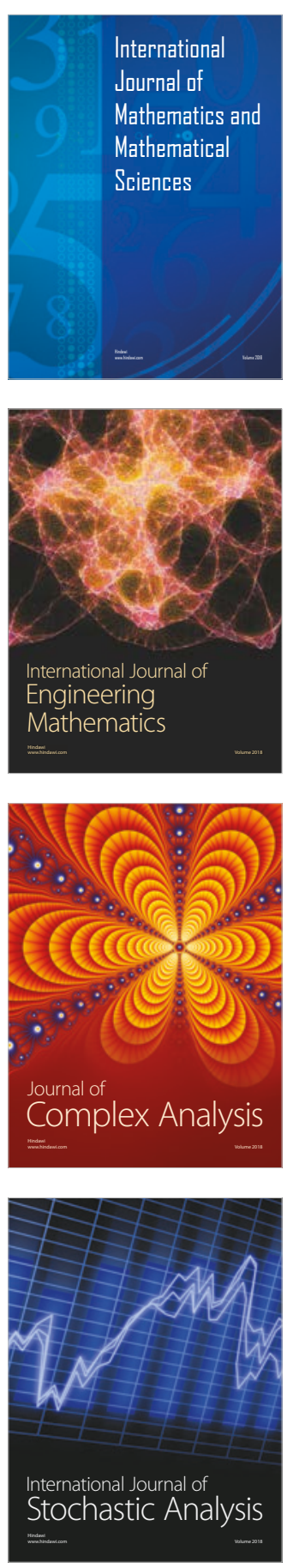
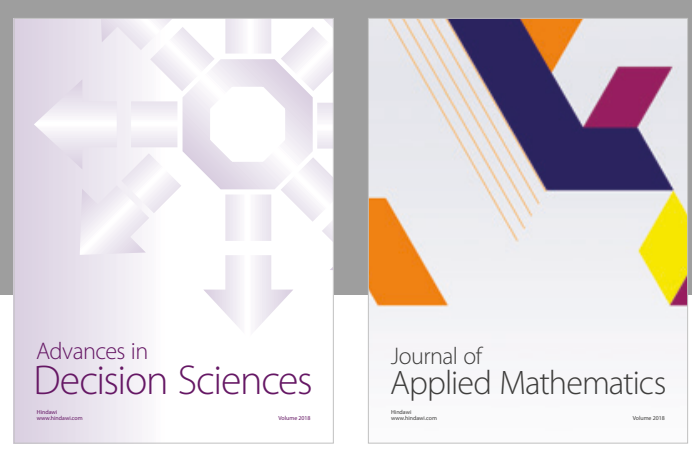

Journal of

Applied Mathematics
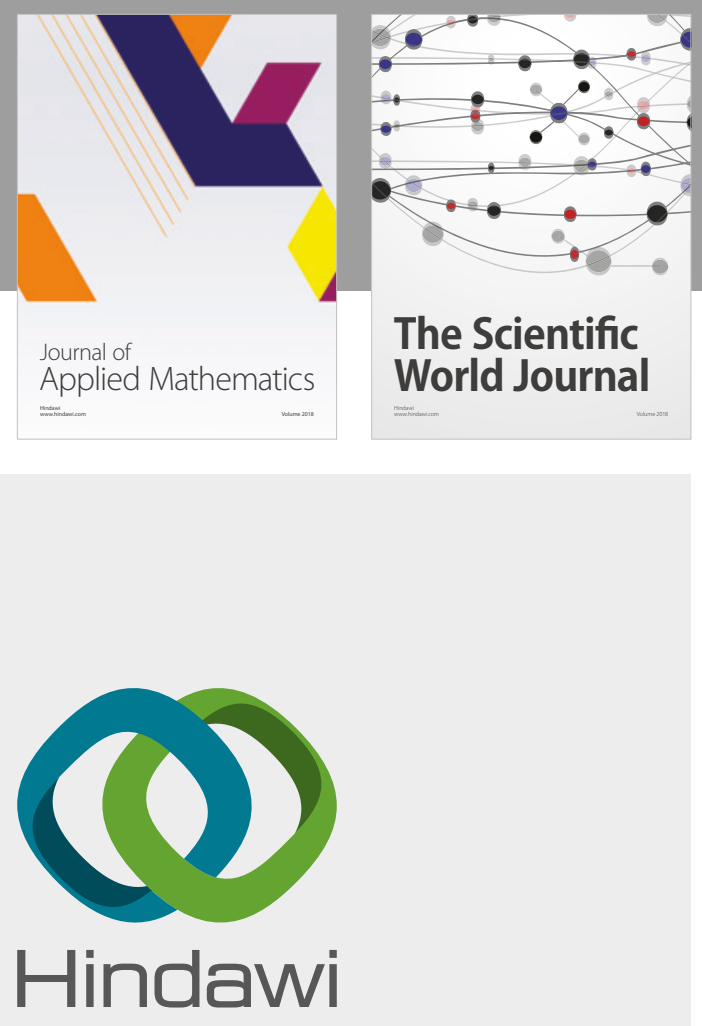

Submit your manuscripts at

www.hindawi.com

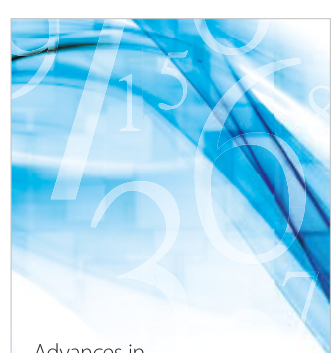

Advances in
Numerical Analysis
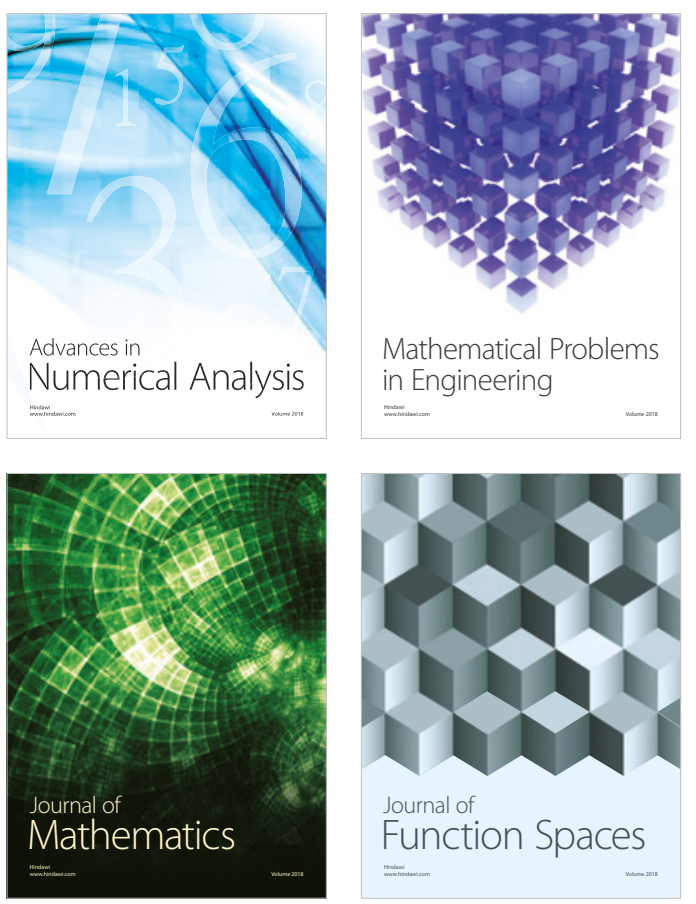

Mathematical Problems in Engineering

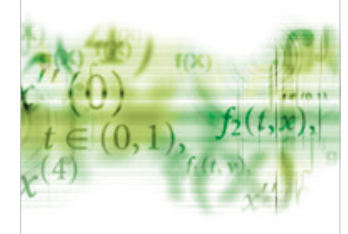

International Journal of

Differential Equations

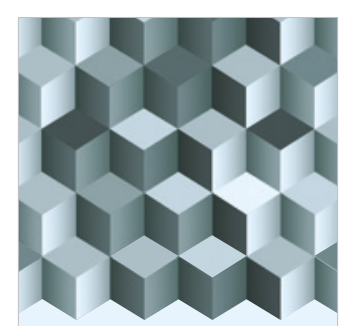

Journal of

Function Spaces
The Scientific

World Journal

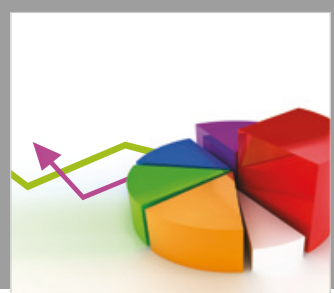

Journal of

Probability and Statistics
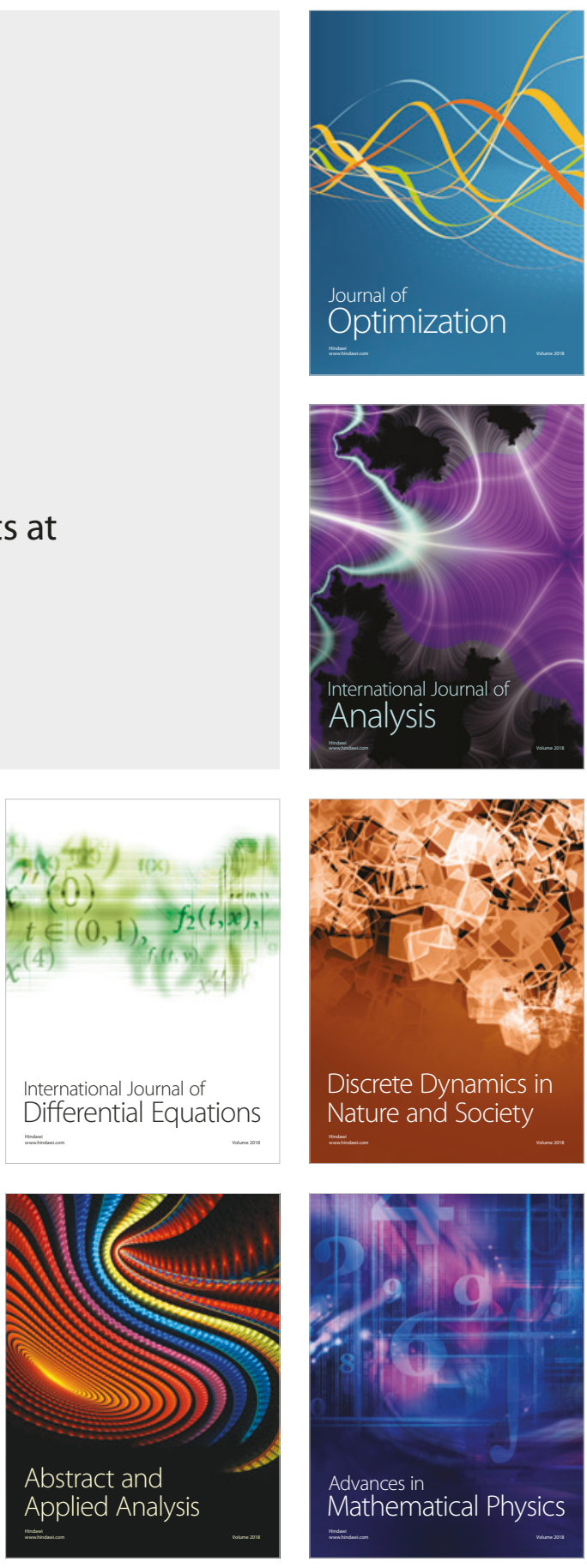